\title{
Processing 3D-Scans Using Statistical Shape Analysis and Automatic Pose Correction for Subsequent Orthosis Fitting
}

\author{
Max THALMEIER, Khoi LAM, Max SCHNAUBELT, Felix GUNDLACK* \\ Mecuris $\mathrm{GmbH}$, Munich, Germany \\ DOI: $10.15221 / 18.010$ http://dx.doi.org/10.15221/18.010
}

\begin{abstract}
In the medical field, 3D-technology enables the creation of individualized medical devices that are tailored to perfectly fit the patient's anatomy. After the acquisition of the patient's 3D-scan, the data needs to be processed before it can be used to design medical devices. Two of the biggest challenges in processing the 3D-data are patient posture and scan quality, where surface information is distorted by noise or foreign bodies. Automatic patient posture correction can be done in numerous ways, but utilizing a generic template model has several advantages. First of all, the template posture can be set to a particular position by the user, reflecting the therapy administered beforehand. The patient scan will then simply match the posture of the model. Additionally, the position of anatomical features of the patient scan can easily be identified with the help of the template model. Another issue needed to overcome is alternating scan quality, which can dramatically decrease the ability to closely fit an orthopedic aid to the patient scan. With the help of machine learning via statistical shape models (SSM), an algorithm can be trained from a dataset of 3D-scans to reconstruct the mesh without affecting the geometrical features of the patient. Afterwards, the repaired and corrected scan can be used to design and print a custom-made orthopedic aid such as an ankle-foot orthosis (AFO).
\end{abstract}

Keywords: 3D-scanning, statistical shape models, automatic pose correction, orthoses, iterative closest point (ICP), template model fitting

\section{Introduction}

In the medical field, 3D-technology enables the creation of individualized medical devices that are tailored to perfectly fit the patient's anatomy. After the acquisition of the patient's 3D-scan, the data needs to be processed before it can be used to design medical devices. Two of the biggest challenges in processing the 3D-data are patient posture and scan quality, where surface information can be distorted by noise.

Tackling the problem of acquiring correct posture, automatically fitting a generic template model, consisting of a skin and bone model from an average person into the patient's 3D-scan has several advantages. First and foremost, the geometry of the scan will be completely "understood", meaning that the position of every bone, joint and anatomical landmark will become known. Additionally, the bone model can be rigged with great detail to physiological functionality which leads to an anatomically accurate corrected scan.

An approach to overcome the secondly described issue of low scan quality is to make use of machine learning to register and make sense out of the 3D-data. From a training set containing various shapes of the anatomies of interest, the training data will first be aligned, which is achievable via Generalized Procrustes Analysis (GPA) [3]. After the alignment process, the learning of shape information can be carried out using Principal Component Analysis (PCA). The result will be a set of basic functions that represent the shape information of the training data. Using these basic functions allows for the creation of a morphable model of specific anatomies (Fig.1), which is also commonly called Statistical Shape Model (SSM) [4]. The entire process chain is displayed in Fig.2. 

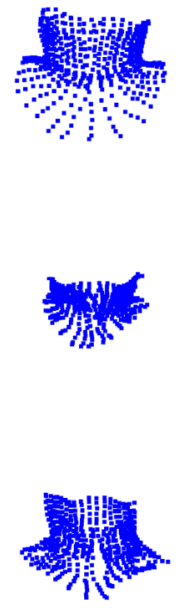
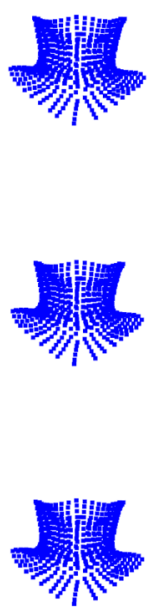
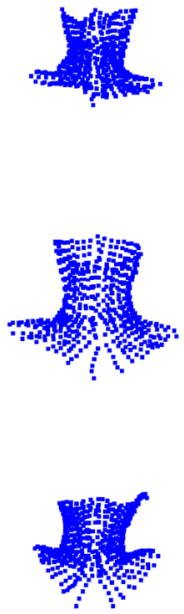

Fig. 1: Various neck models morphed using basic functions obtained via statistical shape analysis.

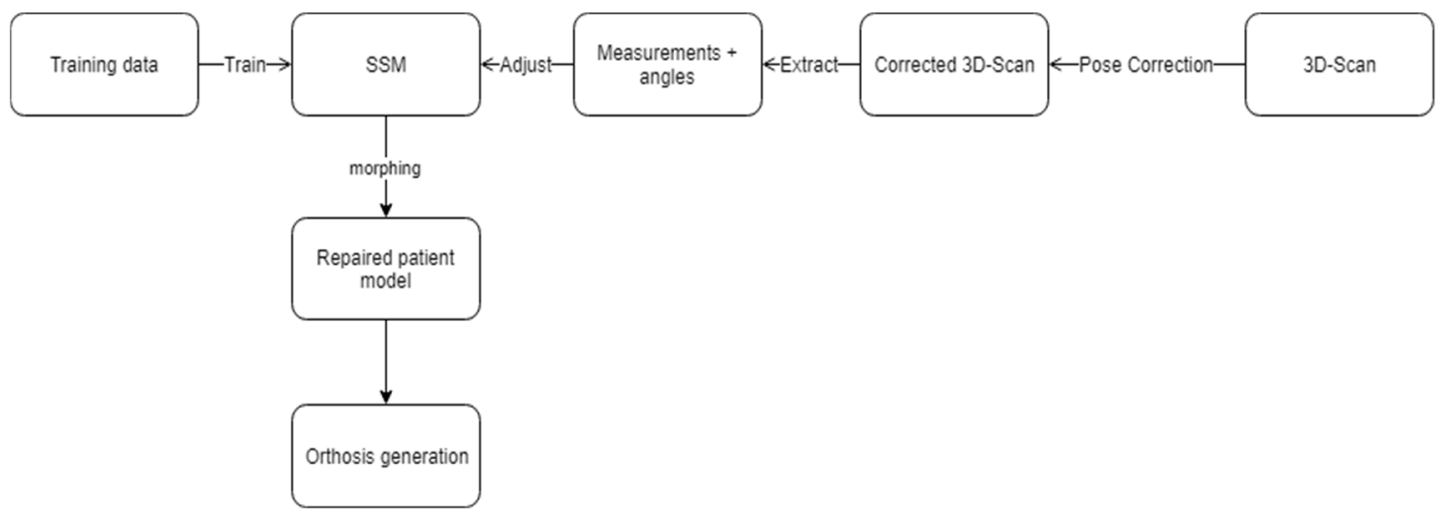

Fig. 2: Block diagram for 3D scan processing and pose correction.

\section{Methods and Materials}

\subsection{Material}

\subsubsection{Data for Pose Correction}

For the testing and validation of the following method of pose-correction, a total of 21 lower-limb 3Dscans were acquired from three different subjects. The scans were obtained with two different 3Dscanners: The Artec Eva [6], a high-end active light scanner and the Occipital Structure [5], which is mounted to an iPad and uses ambient light to generate a 3D-image. Scans were taken in different postures such that the ability of the method introduced in this paper to nullify rotation angles at the ankle joint could be tested with varying lower-limb angles.

\subsubsection{Data for SSM}

The training data used in this study is obtained from Computed Tomography (CT) from the University Clinic of Munich. This dataset contains 128 samples in DICOM format (which is a standard format to store medical images). In order to make use of the CT data set to train the SSM, the first step is to extract the 3D mesh from the DICOM files. This segmentation process can be accomplished by using Imfusion Suit (Imfusion $\mathrm{GmbH}$, Munich).

Besides the training 3D data, test samples are going to be acquired to test the robustness of the generated SSM. The test samples are obtained from 2 different test subjects, each test subject will have 5 different poses, which can be seen in Fig.3. 


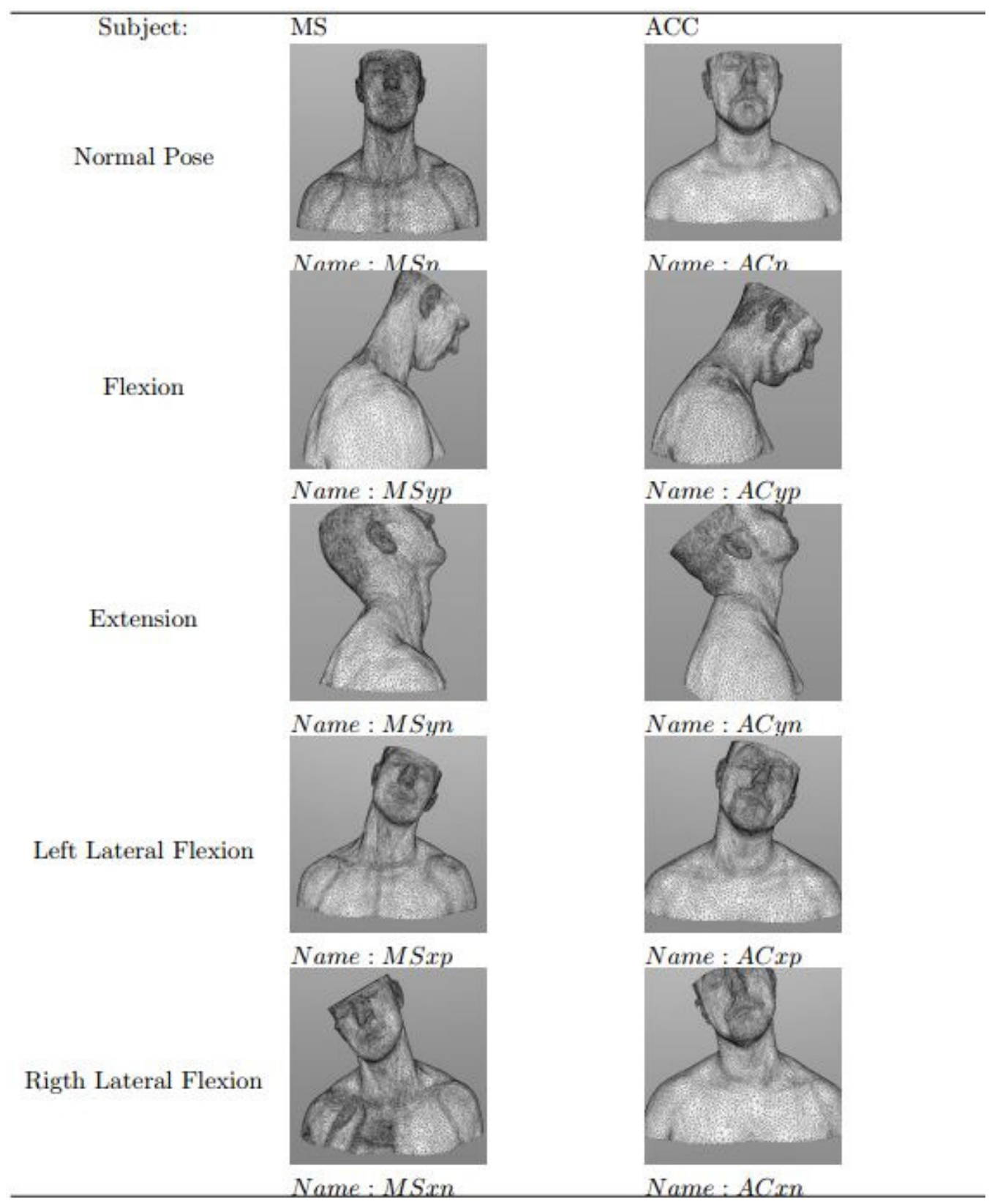

Fig. 3: 10 test scan obtains from 2 test subjects with difference posture.

\subsection{Method}

\subsubsection{Method for pose-correction}

Scan preparation: Before the actual scan processing can begin, a couple of steps are necessary in advance. First, each scan needs to be cleaned, thereby removing any unwanted geometries and small particles from the file. Due to objects in the field of view during scanning, holes might appear in the mesh of the scan and need to be repaired.

Template preparation: The first step is to rig the template. That means the generic template needs to be coupled to an armature that consists of multiple segments and centers of rotation. Luckily, this needs to be done only once and can be reused for every scan. If necessary, the weights of the skinning function can be manually adjusted. 
Template fitting: When the 3D-scan is loaded into the processing software, it is placed according to the position it was acquired based on the coordinate system of the scanner. Therefore, it does not align with the template model at all. As can be seen in Fig.4b, the scan is rotated into the template model. Now, the anatomical landmarks and the Iterative Closest Point (ICP) algorithm will be utilized. The ICP iteratively finds the optimal transformation to align the scan with the skin-model of the template by minimizing the distance between the markers from both objects. As displayed in Fig.4b, a rough fitting of both geometries can be achieved. Additionally, the patient scan is now aligned in the coordinate system of the template model. The next step is now to align the size of both objects. To realize a resizing of the template model, an implementation of the Scaling Iterative Closest Point (SICP) algorithm is used to find scaling parameters in all three dimensions. Similarly to the ICP algorithm, the SICP iteratively calculates a scaling matrix that minimizes the distance between points from both geometries. When the distance between iterations approaches a minimum, the final scaling matrix is accepted and applied to the skin-model as well as the bone-model (Fig.4c). After the rigid resizing of the template model, the ICP implementation is used again to align only the foot of the scan and the template (Fig.4d). As a last step of the template alignment, a vector is calculated which describes the rotation between the tibial part of the template and the scan. This information is subsequently passed to the armature, coupled to the template. Finally, rotating the tibial part of the template along this rotation vector keeps the alignment of the foot section from the previous step and additionally introduces a tibial rotation of the template to match the pose of the scan, visible in Fig.4e.

Pose-correction: When the alignment of patient scan and skin and bones of template model is done, the last remaining step is to apply the rotation vector with a negated rotation angle from the previous step to the armature, which is now coupled to the scan. By doing so, the non-optimal posture of the scan can be corrected by matching the neutral posture of template model $(F i g 4 f+g+h)$.

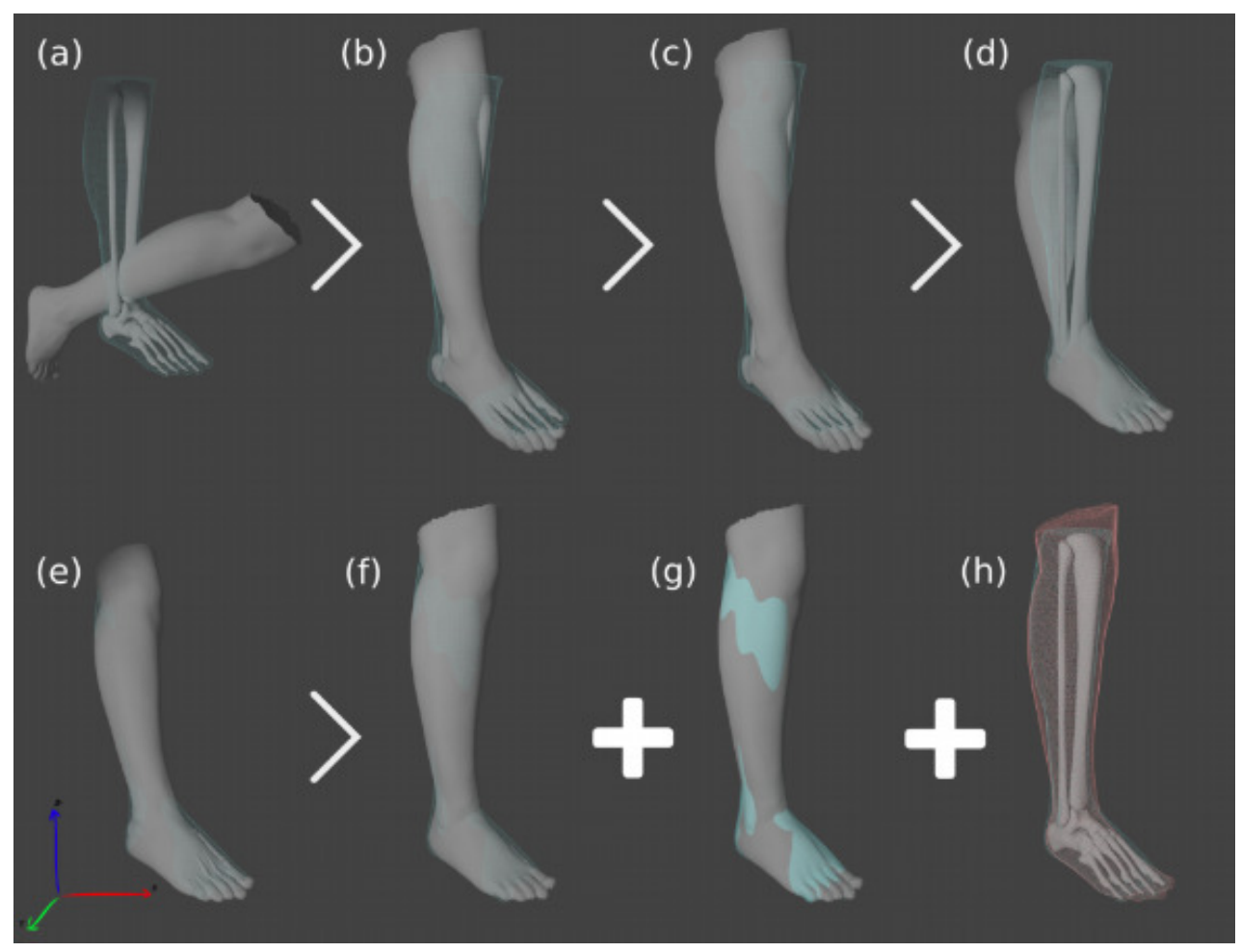

Fig. 4: Overview of the scan processing chain. The skin-model of the template is rendered as a blue wire-frame image showing the underlying bone-model. The prone solid model is the subject's scan. (a) The 3D-scan loaded into Blender does not align with the template model. (b) A first ICP usage roughly aligns both models. (c) The SICP algorithm rescales the template model to better match the scan. (d) Alignment of only the foot-section of both models via another ICP usage. (e) Rotating the tibial part of the template model via the incorporated armature, thereby matching the posture of the scan. (f) Recoupling the armature to the scan and using the inverse rotation of the previous step to rotate the scan into the neutral position of the template model. $(g)$ Different render of the final result shows the skin-model of the template as a solid blue render. (h) Another render of the final result showing the scan as a red wire-frame image with the underlying bone-model. 


\subsubsection{Method for training the SSM}

The majority of the methods [4] used to create the SSM can be described in three steps. First of all, the correspondence of the data in the training shape is established. Next, the registration base on procusted analysis will be carried out, so that we can acquire true shape information (shape information independent of translation and rotation). Finally, the shape information can be extracted from the aligned training set, which is subsequently used as a basis for the statistical shape model.

In order to obtain the correspondence between the samples, a set of 8 landmark will be used to mark the neck/interest region. However, the anatomical landmarks alone is not sufficient for capturing the geometry of the neck/interest region. As a result, a number of Pseudo landmarks is going to be generated in the region between the anatomical landmarks. The anatomical landmarks and the pseudo landmarks is going to be combined into point distribute model (PDM) of the original samples.

After obtaining the PDM for every sample in the training set, the sample data is going to be aligned using GPA. The procrustes analysis relies on having the same number of corresponding points between shapes. There are two modes of procrustes analysis, the rigid mode and the non-rigid mode. The non-rigid procrustes analysis will consider translation, rotation and scaling. On the other hand, the rigid procrustes analysis only considers translation and rotation. In the case of this work, the size of the object is also an interesting parameter. Therefore, a rigid procrustes analysis will be used. An iterative scheme for procrustes analysis can be summarized as follows:

1. Align each sample w.r.t their centroids.

2. Choose one sample to be the initial estimate of the mean shape.

3. Align all samples with the mean shape.

4. Re-estimate the mean shape from aligned samples. If the estimated mean shape doesn't change significantly. Exit the iteration loop, otherwise repeat from step 3.

Finally, we can extract the shape information from the model. To simplify the problem, the dimension of the data should be reduced. A common method is to apply the Principal Component Analysis (PCA) to the training set. The PCA projects the original point onto its principal axes. The process of applying PCA can be described with the following steps [4]:

1. Compute the mean of the data, which can already be obtained via the GPA.

2. Compute the covariance of the data:

$$
S=\frac{1}{s-1} \sum_{i=1}^{s} \quad\left(x_{i}-\underline{x}\right)\left(x_{i}-\underline{x}\right)^{T}
$$

3. By solving the above equation, we can compute the eigenvectors, $\phi i$ and the eigen value $\lambda \mathrm{i}$. If $\phi$ contains T eigenvectors corresponding from the largest eigenvalues to the smallest, then the shape $\mathrm{x}$ can be approximated as follows:

$$
x \approx \underline{x}+\Phi b
$$

where $\Phi=\left(\lambda_{1}, \lambda_{1}, \ldots, \lambda_{t}\right)$ and $\mathrm{b}$ is the parameter vector which has $N-1$ components $(\mathrm{N}$ is the number of training samples).

By varying $b$, we can create a different shape using equation (2), and by limiting $b$ between $\pm \sqrt{\lambda_{i}}$ we can make sure that the generated shape is similar to that in the training shape [4].

In order to fit the created model to a given shape, we could move each model point along its normal to the boundary of the given shape. [5]

From the equation $x \approx \underline{x}+\Phi b$, if we move the model point to fit the target shape we can have the following equation:

$$
x+d x \approx \underline{x}+\Phi(b+d b)
$$

Subtracting both equations from each other, we can obtain the formula to compute shape parameter $b$ :

$$
d b=\Phi^{T} d x
$$




\section{Results}

\subsection{Pose-correction}

The results regarding the automatic pose-correction method described earlier are displayed in Fig.5 and Fig.6, where the corrected posture is split into three separate rotation angles around the ankle joint. As can be seen, the results for correcting plantar- and dorsiflexion angles are exceedingly good. For all three subjects used, the maximum plantar flexion angle after correction was just above $5^{\circ}$ with an overall mean value of $1.7^{\circ}$. Correcting inversion and eversion angles also displayed satisfying results. Corrected angles are overall very low with a mean value of $1.0^{\circ}$, but exhibiting larger deviance. Similarly, the correction of abduction and adduction angles resulted in a low mean value of $2.3^{\circ}$ but high variances.

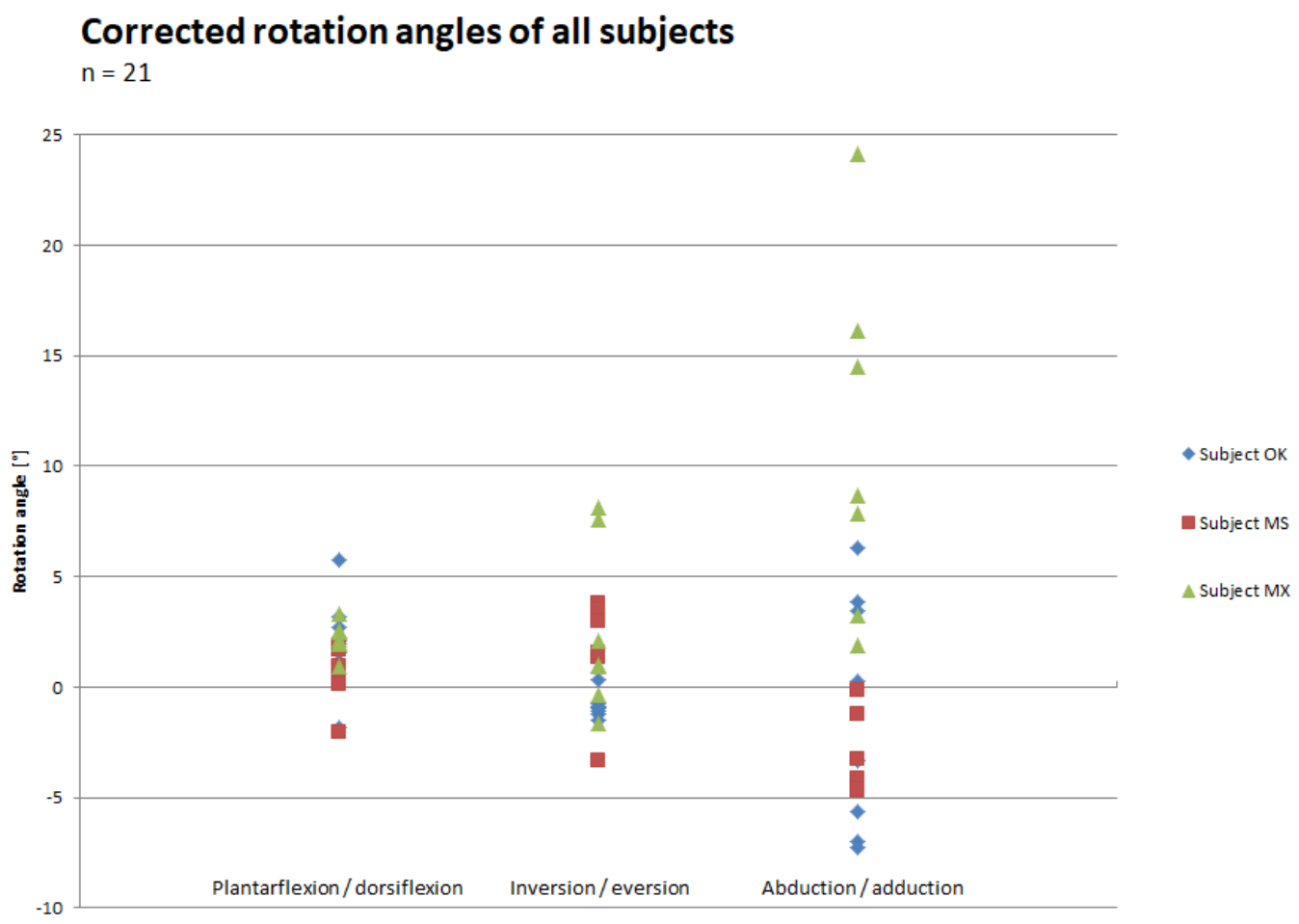

Fig. 5: Results of the automated pose-correction process. Corrected rotation angles are split into three different rotations axes at the ankle joint..

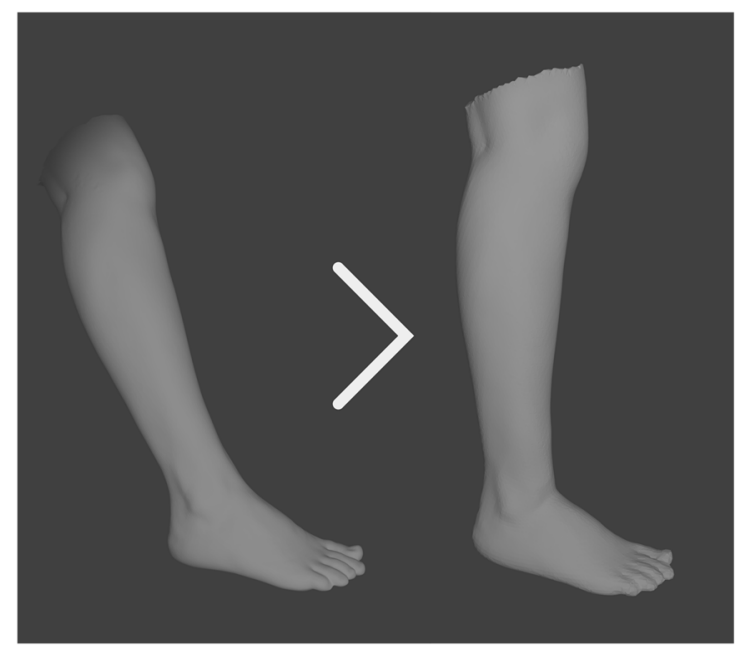

Fig. 6: 3D-scan before and after applying the automated pose-correction process. 


\subsection{Statistical shape Model}

Table 1: Fitting error of the difference test data to the SSM.

\begin{tabular}{|c|c|c|}
\hline Sample Name & Average Error $[\mathrm{mm}]$ & Maximum Error $[\mathrm{mm}]$ \\
\hline ACn & 1.2034 & 5.0843 \\
\hline ACxn & 1.7591 & 8.3611 \\
\hline ACxp & 1.9755 & 6.2905 \\
\hline ACyn & 2.2052 & 10.6309 \\
\hline ACyp & 1.3051 & 5.1830 \\
\hline MSn & 2.8648 & 10.8350 \\
\hline MSxn & 3.1115 & 12.1637 \\
\hline MSxp & 2.9863 & 8.7407 \\
\hline MSyn & 2.9862 & 11.4421 \\
\hline MSyp & 2.1957 & 8.2992 \\
\hline
\end{tabular}

First of all, as can be seen from table, the reconstruction in general yields a better result in case of a normal posture scan (ACn, MSn). Out of all of the pose in the test dataset, the one that has a "flexion" pose (ACyp, MSyp) has the lowest average error as well as maximum error. This result is contributed by the fact that the majority of samples from the training set obtained from the CT-Data are from patients that already have a neck injury. As a result, most of the neck is leaning forward. This leads to the fact that these "flexion" pose scans (ACyp, MSyp) have an error value almost as good as the normal posture case. Finally, one observation regarding the error values is that the reconstruction from test subject $A C$ have a better overall fitting. This result can also be explained by the training data set, which contains mostly elderly and obese patients, who have a smooth and featureless neck shape. Meanwhile, test subject AC has smoother and a non-muscular neck compared to the very muscular neck of test subject MS. Therefore, test subject AC's neck can be reconstructed more accurately using our training dataset.
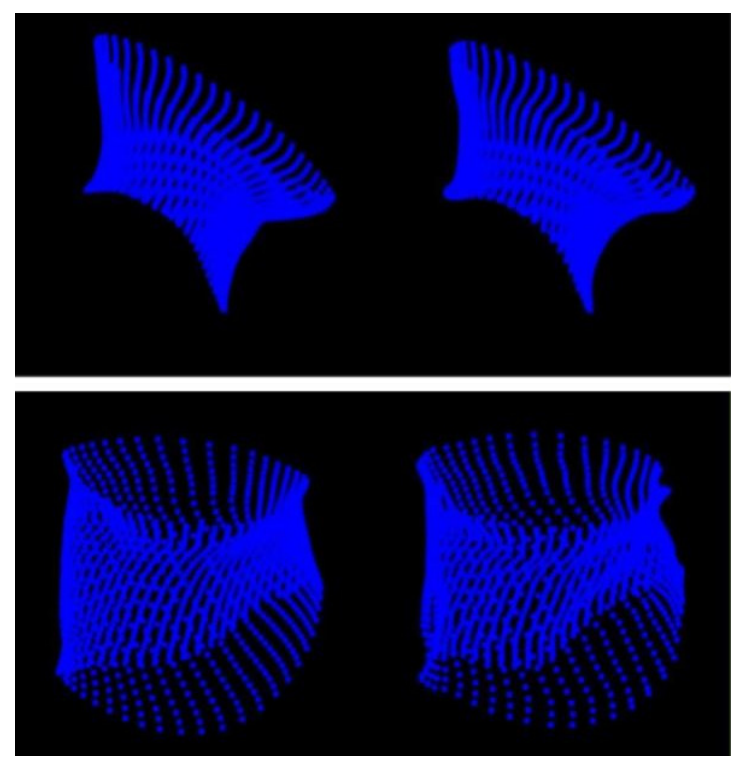

Fig. 7: Visualize the result of the reconstruction. (left is the original, right is the reconstructed) Upper is the reconstruction for test scan ACn. Lower, reconstruction for test scan MSxp. 
Secondly, We can also visually inspect the reconstruction result. Overall, we are able to reconstruct the test data which looks similar to the original. However, in the case where there is a large error value (Fig.7), we can observe the following main regions where the distortion takes place:

- The transition between the neck and the area under the chin.

- The top and bottom horizontal layer of the neck.

- The side where the neck is bent.

\section{Discussion}

\subsection{Accuracy of Posecorrection}

All in all, the process of automatically correcting the scans posture, yielded highly positive results. To put them in perspective, Chuter et al. [8] evaluated the variability of orthopedic technicians to cast the patients lower-limb in a neutral position. They found that even experienced OT's had a forefoot-torearfoot angle, determined by the angle of the posterior bisection of the calcaneus, of $3.0^{\circ} \pm 5.0^{\circ}$. In this work, we were able to achieve a mean plantarflexion angle of only $1.7^{\circ}$. With this comparison at hand, it can be said that the capability of this method to correct the posture of a lower-limb is at least equal to that of an experienced OT, which can be considered a great achievement.

\subsection{Statistical shape model}

After training the model, $\mathrm{N}-1$ main components are obtained, with $\mathrm{N}$ being the number of samples used for training. By varying the first 3 main components we are already able to create a new form (Fig.1). The new forms present a different shape of the neck, via length and diameter changes.

During the reconstruction of the test data using the trained model, all the scans in the training set could be reconstructed. In the case of the model trained with the whole data set, there is an estimated average error of $2.2 \pm 0.7 \mathrm{~mm}$ for all 10 test samples, even though the reconstruction error is pretty high, in case the goal is to use the reconstructed model for fitting orthoses. However, the training set is obtained from a set of emergency CT-scan where either the test subjects are already under a neck injury or the patient is overweight and the neck shape is abnormal, while all of our test data is obtained from a healthy test subject. Therefore, the large average error is acceptable and proves the robustness of the model.

\section{Conclusion}

In this paper, we have proposed a method to prepare 3D data for orthosis fitting process. Our method allows one to automatically correct the posture of input data, which is not always possible to obtain in real life (i.e: due to a pathological condition). After obtaining the pose-corrected 3D data, a model of the patient can be reconstructed using a robust statistical shape model. The final result is a model of the patient data that has a correct posture, independent of the quality of the input scan. This reconstructed model is now ready for the orthosis fitting process.

As an outlook, we could also use our method to obtain the statistical shape model of an orthosis device, which is better known as a parametric model. With both the patient model and the device model, plus matching parameters, one could completely automate the process of designing and fitting orthoses. 


\section{Bibliography}

[1] Besl, Paul J., and Neil D. McKay. 1992. "Method for Registration of 3-D Shapes." In Sensor Fusion IV: Control Paradigms and Data Structures, 1611:586-607. International Society for Optics and Photonics, https://doi.org/10.1109/34.121791.

[2] Du, Shaoyi, Nanning Zheng, Lei Xiong, Shihui Ying, and Jianru Xue, "Scaling Iterative Closest Point Algorithm for Registration of M-D Point Sets.", in Journal of Visual Communication and Image Representation, Special issue on Multi-camera Imaging, Coding and Innovative Display, Vol.21, No.5, 2010, pp.442-52, https://doi.org/10.1016/j.jvcir.2010.02.005.

[3] I. L. Dryden and K. V. Mardia, Statistical Shape Analysis: With Applications in R. John Wiley \& Sons, 2016.

[4] A. Brunton, A. Salazar, T. Bolkart, and S. Wuhrer, "Statistical Shape Spaces for 3D Data: A Review", in Handbook of Pattern Recognition and Computer Vision, World Scientific, 2016, pp. 217-238, https://doi.org/10.1016/j.cviu.2014.05.005.

[5] T. Cootes, C. Taylor, D. Cooper, and J. Graham, "Active shape models-their training and application", in Computer Vision and Image Understanding, vol. 61, no. 1, 1995, p. 38-59, https://doi.org/10.1006/cviu.1995.1004.

[6] 3D Object Scanner Artec Eva | Best Structured-light 3D Scanning Device, https://www.artec3d.com/portable-3d-scanners/artec-eva., accessed 2018.

[7] Best 3D Scanning Software | Artec Studio - 3D Modeling Software, https://www.artec3d.com/3dsoftware/artec-studio., accessed 2018.

[8] V. Chuter, C. Payne, and K. Miller. "Variability of neutral-position casting of the foot.", in Journal of the American Podiatric Medical Association, 93(1):1-5, 2003 Jan-Feb, 